\title{
Causas y consecuencias en el consumo de drogas: un estudio de casos en estudiantes de bachillerato
}

\section{Drogas en el estudiantado}

Ing. Maitta Rosado $\left({ }^{1}\right)$

María Leonila García ( $\left.{ }^{2}\right)$

Jorge Guillermo Cedeño Meza ( $\left.{ }^{3}\right)$

( $\left.{ }^{1}\right)$ Universidad Técnica de Manabí

( ${ }^{2}$ ) Universidad Técnica de Manabí

( $\left.{ }^{3}\right)$ Universidad Técnica de Manabí

Contacto: Ingermaitta7@gmail.com

Receptado: 21/09/2016

Aceptado: 15/11/2016

\section{Resumen}

Este artículo tiene como objetivo proporcionar una revisión de los principales factores psicosociales relacionados con el consumo de drogas en la adolescencia. Para la puesta en marcha de esta experiencia se analizaron estudios de casos desarrollados con estudiantes que consumían drogas en el Colegio Nacional Portoviejo, así como entrevistas al personal del Departamento de Consejería Estudiantil. Los resultados del cotejo probaron que existe una relación significativa entre consumo sistemático de drogas y algunos factores de riesgo presentes en el entor bano familiar y social de los adolescentes. El presente estudio subraya que en relación a una intervención adecuada, debe existir un fortalecimiento en los agentes sociales, familiares, educativos, institucionales, que intervienen en la formación integral de los jóvenes.

\section{Causes and consequences in drug consumption: a study in students of}

\section{Junior High School}


Palabras clave: adolescencia, adicción, drogas, familia, comunicación intrafamiliar.

\section{Abstract}

This article aims to provide a review of the main psychosocial factors related to drug use in adolescence. For the implementation of this experience were analyzed case studies developed with students who use drugs at the "Colegio Nacional Portoviejo", as well as interviews with the staff of the Department of student counselling. The results of the comparison showed that there is a significant relationship between systematic consumption of drugs and some risk factors present in the family and the social environment of adolescents. The present study stresses that in relation to an appropriate intervention, there must be a strengthening of the social partners, family, education, any social institutions, involved in the integral formation of young people.

Key words: Adolescence, addiction, drugs, family, risk factors.

\section{Introducción}

Ante los cambios operados en el mundo globalizado, la sociedad actual ha sufrido grandes transformaciones en todas las esferas, donde la era de la ciencia, la tecnología y el conocimiento obligan a la comunidad en general a hacer frente con pertinencia a estos grandes desafíos.

Si bien el consumo de drogas ha existido a lo largo de la historia de la humanidad, en los últimos tiempos es evidente la relación directa entre sociedad desarrollada y exceso en el consumo de estas sustancias, convirtiéndose en un importante fenómeno que afecta a todos los estratos socio económicos y en cualquier etapa del desarrollo; de manera especial en la etapa de la adolescencia, donde se 
busca alguna puerta de escape hacia lo placentero, con el fin, en la mayoría de los casos, de evadir una realidad dolorosa y difícil de enfrentar.

De acuerdo a la Organización Mundial de la Salud (OMS 1982), droga de abuso es aquella sustancia de uso no médico con efectos psicoactivos, capaz de producir cambios en la percepción, el estado de ánimo, la conciencia y el comportamiento, susceptible de ser autoadministrada y crear dependencia ya sea física, psicológica, social o cualquiera de ellas (Lorenzo, Ladero, Leza, \& Lizasoain, 2010).

Según el Consejo Nacional de Control de Sustancias Estupefacientes y Psicotrópicas (CONSEP) del Ecuador, los jóvenes consumen primero tabaco, alcohol y marihuana (CONSEP, 2008). Un estudio realizado por el Observatorio Social del Ecuador a estudiantes de colegios privados, públicos y fiscomisionales, indica que los estudiantes consumen drogas en las propias instituciones educativas, estas cifras llegan al $48 \%$ de los y las adolescentes a nivel nacional

Adicionalmente, investigaciones realizadas por diversos organismos revelan que cada día, las cifras aumentan aceleradamente; así, en el informe de Niñez y Adolescencia desde la intergeneracionalidad, Ecuador 2016, que recoge información de una encuesta realizada a 4 mil hogares en el país en el año 2015, donde se detallan más de 150 indicadores que describen condiciones sobre el entorno familiar, educación, protección, salud, participación y felicidad. Según los datos de la encuesta, el $29 \%$ de los y las adolescentes entre 12 y 17 años han visto a algún estudiante del colegio consumiendo drogas. En las zonas urbanas este porcentaje supera (34\%) al área rural (18\%). En la Costa, el 38\% de los adolescentes habría visto usar drogas a algún estudiante, una cifra mayor que en otras regiones. Sobre el acceso a drogas y estupefacientes, un 15\% señala que le sería fácil conseguir marihuana. Lo preocupante, detalla el informe, es que la segunda droga a la que, al parecer, pueden acceder es la $\mathrm{H}$ en un $8 \%$. En tercer lugar está la cocaína con un $6 \%$, el éxtasis en un $4 \%$ y la pasta base en un $3 \%$. 
El estudio indica que lo más grave es que el $46 \%$ considera que en su centro educativo circulan supuestamente drogas y el $24 \%$ dice que alguna vez habría visto a un estudiante que las vende o las pasa. Casi la mitad de los y las encuestadas (48\%) asegura que alrededor de su colegio o escuela venden estupefacientes (Observatorio Social del Ecuador, 2016, pág. 118) Finalmente señala que el trabajo de la sociedad y el Estado tiene que enfrentarse a un mercado que busca entre los más jóvenes a sus 'clientes', para acostumbrarlos al consumo y asegurar el futuro de las ventas, sin importar los daños en distintos aspectos de la vida de estos jóvenes, que eso conlleva.

Teniendo en cuenta estas dificultades de la realidad mundial y de manera específica del Ecuador, este trabajo se encuentra fundamentado hacia el objetivo de analizar el consumo de drogas, en casos específicos de estudiantes de una institución educativa de la ciudad de Portoviejo, sus causas y consecuencias así como la intervención de los diferentes agentes educativos.

\section{Desarrollo}

\section{El adolescente y el consumo de drogas}

La literatura consultada revela que detrás de una conducta, siempre hay una persona producto de una sociedad y de sus interacciones entre las condiciones psicosociales y la cultura elaborada por ella misma en cada momento de la historia. En este trabajo se analiza la conducta de esa persona en la etapa de la adolescencia; considerando que esta es una etapa de la vida en la cual los jóvenes se encuentran en camino de la búsqueda de su identidad, de la lucha por alcanzar su independencia y de un modelo sobre el cual proyectar su vida, esto ocasiona en muchos casos el rechazo a una escala de valores propuesta por sus padres o la sociedad y sus instituciones en general.

No es de sorprender que la adolescencia sea la época más frecuente de experimentación con las drogas incluyendo el tabaco y el alcohol. Son muchos los factores que intervienen para ello: su familia, su grupo de amigos, su 
comunidad...; es decir, este adolescente no debe ser estudiado como un ente solitario, sino inserto en una realidad espacial y temporal, que a su alrededor se encuentra su familia y su contexto, que no puede ser diagnosticado en un corte vertical de su vida, porque tiene una realidad transversal con un pasado y un futuro (Urra, 2003), aspecto confirmado por (Rossi, 2008, pág. 11) cuando indica que las drogas son un problema de todos: de quienes consumen, de las familias, de los adictos, de las asociaciones intermedias, de las entidades religiosas, de la comunidad médica, de la salud pública y privada, de los docentes y del Estado. $Y$ de todas aquellas entidades públicas o privadas que tienen como responsabilidad el aseguramiento de la calidad de vida a través de los bienes y o servicios que ofrezcan.

Por otro lado, no por ser menos importante, es necesario considerar los esquemas cognitivos, los que podrían actuar como factor de riesgo para el consumo de drogas, especialmente cuando se combinan con un estilo impulsivo de resolución de problemas, al igual que se ha demostrado para otras conductas antisociales (Calvete \& Estévez, 2009) aspecto que debe ser analizado con detenimiento y profunda responsabilidad,

Para conocer a mayor profundidad cuál es la conducta del adolescente que consume drogas y en qué fase de adicción está, resulta importante saber que al igual que en las demás conductas humanas, en el consumo de drogas pueden observarse unas secuencias de desarrollo bien definidas; sin embargo, la formulación de estos estadios no implica que el sujeto que consuma una determinada sustancia debe necesariamente consumir la siguiente, sino más bien que la mayoría de los sujetos que se encuentran en una determinada etapa han consumido las sustancias que conforman las fases anteriores. El consumo de una droga situada en los primeros estadios es una condición necesaria, pero no suficiente para la progresión a un estadio posterior. (Gonzalez, García, \& Gonzalez, 1996, pág. 258), datos significativos que permitirán una intervención mucho más efectiva.

En concordancia a lo anterior, de acuerdo a (Cornide, Ruiz, Torres, \& Alcaraz, 2002, pág. 82) resulta importante distinguir dos clases de consumidores de 
drogas: el consumidor experimental, que es aquel que prueba la droga una 0 más veces en su afán de conocer nuevas sensaciones, pero no suele volver a consumirla; y el consumidor sistemático a la forma de consumo habitual que caracteriza a la drogodependencia o lo que se conoce como adicción, que no es otra cosa que un grupo de fenómenos fisiológicos, conductuales y cognitivos de variable intensidad, en el que el uso de sustancias psicoactivas tienen una alta prioridad; se trata entonces de una conducta obsesiva compulsiva que lleva a consumir periódicamente la droga para experimentar un estado afectivo positivo como el placer, bienestar, euforia, sociabilidad, escape de la realidad, búsqueda o abandono de la identidad, exploración de nuevas sensaciones 0 librarse de un estado negativo como el dolor, aburrimiento, timidez, estrés, preocupaciones, decepciones, entre otros.

Frente a esta realidad de los jóvenes, no solo de Ecuador, sino del mundo entero, muchos han sido los esfuerzos en todos los niveles para lograr reducir en algo la situación; sin embargo los resultados no se ven disminuidos, los esfuerzos se vuelven débiles, ya que la mayoría trabaja sólo o de manera aislada; los padres de familia, por un lado, en su desesperación por hacer algo, o por su intervención tardía, y por otro, los maestros que cada vez más se sienten disminuidos en su gestión por las leyes que sobreprotegen a los niños y jóvenes y por la ausencia de los padres o la familia en el desarrollo de los mismos, siendo recomendable que desde las instituciones se promueva un acercamiento para incidir sobre los jóvenes de forma coordinada y complementaria (Rodríguez, 2015, pág. 182)

\section{Las políticas frente al consumo de drogas}

La convención sobre los Derechos del Niño (UNICEF 2006) en el Artículo 27.nำ1, indica que "Los Estados Partes reconocen el derecho de todo niño a un nivel de vida adecuado para su desarrollo físico, mental, espiritual, moral y social" ratificando de esta manera el accionar que todo niño o niña tiene derecho a beneficiarse de un nivel de vida adecuada para su desarrollo integral y es responsabilidad primordial de padres y madres proporcionárselo. A más de ello, es obligación del Estado adoptar medidas apropiadas para que dicha responsabilidad pueda ser asumida. 
Por otra parte, y para hacer frente al problema sobre el consumo de drogas a nivel mundial; la literatura nos revela que en el contexto del Sistema de las Naciones Unidas, existen tres instrumentos internacionales sobre la fiscalización internacional de drogas, a saber: la Convención Única de 1961 sobre Estupefacientes enmendada por el Protocolo de 1972; el Convenio sobre Sustancias Sicotrópicas de 1972; y la Convención de las Naciones Unidas contra el Tráfico llícito de Estupefacientes y Sustancias Sicotrópicas de 1988. Los órganos internacionales consagrados en materia de fiscalización y seguimiento son la Junta Internacional de Fiscalización de Estupefacientes (JIFE), el Programa de las Naciones Unidas para la Fiscalización Internacional de Drogas (PNUFID) y la Comisión de Estupefacientes del Consejo Económico y Social de Naciones Unidas (ECOSOC).

El tema de las drogas también ha tenido fuerte presencia en las Cumbres presidenciales hemisféricas a lo largo de la década de los noventa. Así, en la Cumbre de Miami, en 1994, se planteó la construcción de un sistema integral y consensuado de derechos y responsabilidades para enfrentar el consumo indebido de drogas y los problemas conexos. Y en la Cumbre de Santiago, en 1998, se adoptó un Plan de Acción para los próximos años, y un sistema de evaluación imparcial y técnica de los avances del mismo, bajo la dirección de la CICAD, la Comisión Interamericana para el Control del Abuso de Drogas, de la Organización de Estados Americanos (OEA).

La Constitución de la República del Ecuador (2008) en el Art. 3 numeral primero establece como deber primordial del Estado "Garantizar sin discriminación alguna el efectivo goce de los derechos establecidos en la Constitución y en los Instrumentos Internacionales, en particular la educación, la salud, la seguridad social y el agua para sus habitantes", norma constitucional que se considera, ya que se reconoce a la drogadicción como una enfermedad que consiste en la dependencia del ser humano a una de las sustancias psicotrópicas y estupefacientes que existen en el mercado, mismas que afectan especialmente al sistema nervioso central y las funciones cerebrales. 
En relación al consumo de drogas, esta misma constitución en el Art. 364 determina que "Las adicciones son un problema de salud pública. Al Estado le corresponderá desarrollar programas coordinados de información, prevención y control del consumo de alcohol, tabaco y sustancias estupefacientes y psicotrópicas; así como ofrecer tratamiento y rehabilitación a los consumidores ocasionales, habituales y problemáticos, en ningún caso se permitirá su criminalización ni se vulnerarán sus derechos constitucionales"

La Constitución de la República establece varios aspectos fundamentales que encauzan con amplitud y profundidad la necesidad de que la prevención se realice desde una visión holística e integral, orientada de manera prioritaria hacia niñas, niños, adolescentes y jóvenes. Concibe las adicciones como un problema de salud pública y consecuentemente preveé la intervención del Estado y de la comunidad en el desarrollo de programas de prevención, tratamiento rehabilitación e integración social y laboral, aspectos estos de orden multidimensional que se hallan formulados en la estrategia nacional de Prevención Integral y control de Drogas para el próximo cuatrienio, en cuya gestación y coordinación técnica intervino el Consejo Nacional de Control de Sustancias Estupefacientes y Psicotrópicas, CONSEP.

\section{Métodos y técnicas}

Para el desarrollo de la experiencia, se realizó una profunda revisión bibliográfica especializada, así como el análisis de estudios de casos desarrollados con estudiantes que consumían drogas en el Colegio Nacional Portoviejo, entrevistas al personal del Departamento de Consejería Estudiantil. También fue de mucha importancia la observación directa de acciones emprendidas en la institución, frente a situaciones parecidas a los casos analizados.

\section{Resultados y discusión}

\section{CASO № 1}

DATOS GENERALES DE LA ESTUDIANTE

\section{Edad 16 años}

Características del hogar Disfuncional 


\begin{tabular}{r|l} 
Motivos de consumo & Violencia intrafamiliar \\
Edad inicial de consumo & A los 15 años \\
Droga consumida por la menor & Marihuana, alcohol \\
Nivel económico familiar & Bajo \\
Nivel educacional de la madre & Primaria \\
Nivel educacional del padre & Primaria \\
No de personas con las que vive & 4 (mamá y 3 hermanos) \\
Ayuda y Orientación dentro del Colegio & Si \\
\hline Fuente: $\quad$ Registros del Colegio Nacional Portoviejo \\
Elaboración: $\quad$ Investigadoras
\end{tabular}

El caso número uno corresponde a una joven de 16 años, vive con la mamá y tres hermanos; desde muy temprana edad ha sufrido violencia intrafamiliar donde su padre alcoholizado propinaba fuertes golpes a la madre. La relación con la madre es de poca comunicación, incluso de serias confrontaciones. Manifiesta estar cansada de los problemas en su hogar; los fines de semana acude a fiestas y discotecas, no le importa si no le dan permiso, igual se escapa, le tiene sin cuidado si la castigan o no. Inició su experiencia bebiendo cervezas, hasta que alguien le brindó un "pito" de marihuana, lo aceptó con temor, pero la sensación de olvidarse de lo que le sucedía, la "transportaba" hizo que continuara con su consumo.

En sus inicios recibía la droga como obsequio de sus amigos, quienes para obtenerla le robaban a sus padres; luego ha tenido que conseguir dinero vendiendo sus pertenencias (celular, maquillaje, carteras, otros) hasta ahora sólo ha consumido marihuana porque "es más barata". En cuanto a la manera de conseguir la droga, manifiesta que es muy fácil ya que "está como en las panaderías", es decir en cualquier lugar .Considera que es mala estudiante, ya que asiste al colegio porque su madre la obliga, no tiene aspiraciones profesionales por lo que no le importa en lo más mínimo si pierde o no el año escolar.

En las entrevistas con la progenitora, evidencia insatisfacción por el tipo de vida que lleva: por la situación económica, por la falta de trabajo, por la crianza de los hijos y la decepción que estos le ocasionan. 
Como parte de la intervención del Departamento de Consejería Estudiantil, la trabajadora social manifiesta en su informe que el nivel de comunicación de los miembros de la familia es deficiente. La orientadora desarrolló un programa de apoyo mediante entrevistas personales de manera periódica, y abordó temas específicos como de autoestima. Con el apoyo y seguimiento mostró un ligero cambio de comportamiento; sin embargo la familia muy poco colabora en el mejoramiento de la joven, quien manifiesta por momentos deseos de cambio, pero no es constante, por lo que resulta imprescindible un programa de apoyo familiar que promueva el interés por la situación personal y académica de la estudiante y trabajar en equipo, ya que se puede asegurar que desde siempre se reconoce a la familia y a la escuela como los dos sistemas más influyentes en el desarrollo del individuo (Rodríguez, 2015)

\section{CASO № 2}

\section{DATOS GENERALES DE LA ESTUDIANTE}

\section{Edad 17 años}

Características del hogar

Motivos de consumo

Estructurado

Influencias sociales negativas

Edad inicial de consumo

Droga consumida por la menor

Nivel económico familiar

Nivel educacional de la madre

Nivel educacional del padre

№ de personas con las que vive

Ayuda y Orientación dentro del Colegio

5 meses antes de cumplir 17 años

Marihuana

Medio

Secundaria incompleta

Secundaria

Mamá, papá y 1 hermano

Fuente: $\quad$ Registros del Colegio Nacional Portoviejo

Elaboración: Investigadoras

El caso número dos corresponde a una joven de 17 años de edad, reside en la zona urbana del cantón Portoviejo, en un sector considerado "zona roja" por ser muy frecuente la venta de drogas. El sustento del hogar se obtiene de un modesto taller de costura que permite suplir las necesidades de sus hijos, sin descuidar la enseñanza y los buenos principios, según reporte de los progenitores e informe de la Trabajadora Social del Plantel.

Según reporte la adolescente se inicia en este mundo cuando a escondidas de sus padres frecuentaba a un grupo de "amigos de mala reputación a quienes se 
los acusa de consumidores", a partir de aquello abandonaba el colegio antes de terminar la jornada de estudios, se dormía en clases, mostraba siempre rostro cansado, pupilas dilatadas, lo que fue notorio para sus maestros; a ello se le suma la despreocupación continua en las tareas educativas, el prolongado sueño, provocando un bajo nivel en su rendimiento académico.

La institución educativa inicia su intervención identificando el problema tanto de la estudiante como de su círculo familiar, confirmando el consumo de marihuana y de la adicción a la misma; junto a los padres se inicia un trabajo en equipo, quienes con toda la predisposición buscaron ayuda externa de profesionales que pudieran apoyar a la adolescente en su recuperación. Por su parte la orientadora del plantel realiza seguimiento y evalúa constantemente los progresos de la estudiante para lo cual mantiene continua comunicación con los padres, para lograr mejores resultados.

Después de varios meses de intervención, tanto del plantel educativo, como de otros organismos dedicados a este fin, se evidencia mayor control por parte de los padres, la familia ha mejorado en sus niveles de comunicación y lo más importante, la joven ha dejado de consumir drogas y tiene proyectos de vida, entre ellos, pretende graduarse sin ninguna dificultad, evidenciándose en su comportamiento y en su desempeño académico, confirmando de esta manera lo expresado por Arriaga y Hopenhayn cuando manifiestan que una buena política de prevención y control de drogas debe incorporar los instrumentos más consagrados de la política social actual, el uso de la comunicación para lograr los objetivos deseados, y las formas más eficientes para controlar y reprimir el delito en el contexto del Estado de Derecho. Esto implica incorporar criterios de focalización, selectividad, descentralización de la gestión, sinergia, eficiencia y eficacia. (Arriaga \& Hopenhayn, 2000, pág. 36)

\title{
CASO № 3
}

\author{
Edad 16 años \\ Características del hogar Funcional \\ Motivos de consumo Vulnerabilidad por abuso sexual
}




\begin{tabular}{r|l} 
Edad inicial de consumo & A los 14 años \\
Droga consumida por la menor & Marihuana \\
Nivel económico familiar & Bajo \\
Nivel educacional de la madre & Primaria \\
Nivel educacional del padre & Primaria \\
No de personas con las que vive & 4 personas (mamá y papá 2 hermanos) \\
Ayuda y Orientación dentro del Colegio & Si \\
\hline Fuente: & Registros del Colegio Nacional Portoviejo \\
Elaboración: & Investigadoras
\end{tabular}

El caso número tres hace referencia a una adolescente de 16 años de edad, residente en la zona rural de la provincia de Manabí (San Jacinto- cantón Sucre); sus progenitores son personas sencillas dedicadas a la agricultura, convirtiéndose éste en el medio de sustento de la familia.

Como dato importante se registra que a pesar de los cuidados la joven fue abusada sexualmente por un familiar, originando serios problemas en la conducta y personalidad de la adolescente, siendo introvertida y de pocos amigos.

Por la distancia con su lugar de estudios, debe viajar varios kilómetros diariamente, y en uno de sus viajes conoce a una persona mayor que estudia en una universidad local quien le ofrece su amistad y la involucra en el mundo las drogas. El cambio en su comportamiento fue inmediato y notorio, quien a pesar de ser introvertida se caracterizaba por su responsabilidad con las tareas escolares, ya que empezó a llegar atrasada al colegio y a veces faltaba a clases y su despreocupación por los estudios fue aumentado.

Entre las causas que la llevaron al consumo, la joven indica que "en las drogas encontraba una salida a su realidad", el recuerdo del abuso del que había sido víctima y que no lo podía comentar con nadie, la amabilidad y dulzura que encontró en "el amigo" y sentirse sola ya que la comunicación con la familia era mínima. Normalmente los adolescentes presentan diversos grados de vulnerabilidad, frente al estrés, la frustración y la ansiedad, lo que ocasiona que se incremente la probabilidad o el riesgo de involucramiento y desarrollo de ciertos comportamientos adictivos, con y sin sustancias. (CEDRO, Centro de información y educación para la prevención del abuso de drogas, 2013, pág. 33) 
La institución junto a la familia emprende el proyecto de recuperación; los padres en un primer momento se rehúsan y no aceptan la realidad de su hija, pero poco a poco se consigue que asumen la condición actual, se establece convenio con instituciones estatales y particulares para que la adolescente reciba la ayuda necesaria tanto física como psicológica e incluso espiritual, considerando que el problema a resolver no solo era el consumo de drogas sino también el daño psicológico provocado por el abuso sexual del cual fue víctima. Este proceso se llevó a lo largo de todo el año lectivo, se involucró al tutor del curso quien estableció el vínculo entre los docentes y la familia, así como el personal del DECE del plantel y las autoridades que estuvieron pendiente en todo momento.

La condición de la adolescente evidencia mejoría de manera lenta, incluso en algunos momentos parece existir retrocesos en su comportamiento; sin embargo el apoyo de la familia no desmaya y permanecen pendientes de su tratamiento, permitiendo esto que la comunicación con su hija haya mejorado significativamente; por otra parte cuenta con un pequeño grupo de amigos con los que eventualmente sale de paseo y sus calificaciones van en aumento.

\section{Conclusiones}

De acuerdo al análisis efectuado sobre las causas y consecuencias del uso de drogas en estudiantes de bachillerato, mediante el estudio de casos específicos, se concluyó que los adolescentes por estar en una etapa crítica del desarrollo marcada por la búsqueda de identidad, deseo de independencia, entre otras, se constituyen en la población más vulnerable a desarrollar adicciones y que el ambiente familiar ( relación padres e hijos, los estilos de crianza, clima familiar, entre otros), el círculo de amigos y experiencias traumáticas de abuso (sea físico, psicológico, sexual) tienen una correlación significativa en el consumo de drogas. Con respecto a una adecuada intervención primaria resulta relevante el trabajo coordinado entre la escuela, la familia, las instituciones y los profesionales en el área, para que contribuyan al mejoramiento del estilo de vida de los adolescentes y esto derive en una recuperación de su salud física y mental libre de drogas. 


\section{Bibliografía}

1. Arriaga, I., \& Hopenhayn, M. (2000). Producción, tráfico y consumo de drogas en América Latina. Santiago de Chile: División de Desarrollo Social.

2. Barra, A. (2015). Política de drogas en América Latina: obstáculos y próximos pasos. Nueva Sociedad, 81-93.

3. Calvete, E., \& Estévez, A. (2009). Consumo de drogas en adolescentes: El papel del estrés,. Adicciones, 21( 1), 49-56.

4. CEDRO, C. d. (2013). Abuso de drogas en adolescentes y jòvenes y vulnerabilidad familiar. Perú: Oficina de las Naciones Unidas contra la droga y el delito (UNODC).

5. CONCEP. (2008). TERCERA ENCUESTA NACIONAL SOBRE CONSUMO DE DROGAS EN ESTUDIANTES DE ENSEÑANZA MEDIA 2008. QUITO: ONUDD.

6. CONSEP, C. N. (2008). TERCERA ENCUESTA NACIONAL DE SUSTANCIAS ESTUPEFACIENTES $Y$ PSICOTRÓPICAS - CONSEP. ECUADOR.

7. Cornide, A., Ruiz, A., Torres, M., \& Alcaraz, G. (2002). Consumo de alcohol, tabaco y otras drogas en adolescentes, estudio de dos cortes. Medicina de la familia, 81-87.

8. ECUADOR, A. N. (2015). LEY ORGÁNICA DE PREVENCIÓN INTEGRAL DEL FENÓMENO SOCIONECONÓMICO DE LAS DROGAS Y DE REGULACIÓN Y CONTROL DEL USO DE SUSTANCIAS CATALOGADAS SUJETAS A FISCALIZACIÓN. QUITO: ASAMBLEA NACIONAL DEL ECUADOR.

9. ECUADORINMEDIATO. (MARTES de 06 de 2016). ANALISIS DEL PROYECTO DE LEY DE PREVENCION DE DROGAS. RESULTADOS DE CUARTA ENCUESTA SOBRE USO DE DROGAS A ESTUDIANTES DE 12 A 17 AÑOS, pág. EDICION \#4135.

10. GARCÍA, P. (8 de MAYO de 2013). EL CONSUMO DE LAS DROGAS EN ADOLESCENTES. SALUD, pág. 4B.

11. Gonzalez, F., García, M., \& Gonzalez, S. (1996). El consumo de drogas en la adolescencia. Psicothema, 8(2), 257-267.

12. LORENZO, P., LADERO, J., LEZA, C., \& LIZASOAIN, I. (2010). DROGODEPENDENCIAS (TERCERA ed.). MADRID: PANAMERICANA.

13. Observatorio Social del Ecuador. (2016). Niñez y Adolescencia desde la intergeneracionalidad. Offset Abad.

14. Ortega, B. B. (2009). Prevalencia y factores asociados al consumo de drogas en los/las adolescentes de los colegios nocturnos de la ciudad de Cuenca. Cuenca: Universidad de Cuenca.

15. Rodríguez, A. (2015). Formación y desarrollo de la comptencias orientación educativa familiar en los docentes de básica superior. SIPNASIS, 6(1). 
17. 16. Rodríguez, A. (2015). Modelo didáctico para la formaciòn y desarrollo de la competencia "orientar a la familia" en docentes de bàsica superior en ejercicio. Didasc@lia: didáctica y educación, $\mathrm{VI}(6), 181-188$.

18. Rossi, P. (2008). Las drogas y los adolescentes. lo que los padres deben saber. Madrid: TÉBAR.SI.

19. Salazar, I., \& Arrivillaga, M. (2004). El consumo de alcohol, tabaco y otras drogas como parte del estilo de vida de jóvenes universitarios. revista colombiana de Psicología(13), 74-85.

20. Universo, E. (4 de abril de 2016). el 46 \% de jòvenes de Ecuador cree que droga circula en su plantel. Diario el Universo, pág. 24 A.

22. UNIVERSO, E. (4 de ABRIL de 2016). EL 46\% DE JOVENES DE ECUADOR CRRE QUE DROGA CIRCULA EN SU PLANTEL. EL 46\% DE JOVENES DE ECUADOR CRRE QUE DROGA CIRCULA EN SU PLANTEL, pág. $8 \mathrm{~A}$.

23. Urra, J. (2003). Adolescencia y violencia. Tópicos y realidades. Revista de estudios de juventud, 11-20. 